\title{
Signatures of Braking Surface Targets in Spotlight Synthetic Aperture Radar
}

\author{
David Alan Garren \\ Electrical and Computer Engineering Department \\ Naval Postgraduate School \\ Monterey, California 93943, USA \\ Email: dagarren@nps.edu
}

\begin{abstract}
This paper examines the signature characteristics in spotlight synthetic aperture radar (SAR) image data for surface targets that are executing braking maneuvers during the SAR collection. This analysis considers the special case in which the radar sensor is assumed to move with constant speed and heading on a level flight path with broadside imaging geometry. The analysis concentrates on the target migration effects in which the moving target smear exhibits some defocus in the range direction, although much smaller in magnitude than the smearing in the radar crossrange direction. This paper focuses on the case of a target that executes a rapid speed transition by decreasing its speed within the synthetic aperture. The SAR simulations are shown to give signatures that are in agreement with the predicted shapes.
\end{abstract}

\section{INTRODUCTION}

Moving targets cause smeared signatures within synthetic aperture radar (SAR) imagery for all imaging modes. This effect is well-known and has been studied by numerous researchers. For example, Perry et al. [1] develops techniques for generated refocused target signatures based upon the smeared signatures of moving targets. These methods assume that the smearing lies entirely in the radar cross-range direction, as has been assumed with most other studies. In addition, a number of other researchers have studied target migration and moving target signatures within SAR imagery [2], [3], [4]. In particular, Jao [5] has presented the results that moving target signatures can exhibit a basic curved bowing shape, wherein the majority of the smearing still lies in the radar cross-range direction.

Recent studies [6] examines the signature characteristics of moving targets in SAR image data for cases of non-uniform target motion. Specifically, this analysis considers the case in which a target moves with constant heading at a slower speed, then increases its speed to a higher speed for some time, and then decreases its speed back the original speed. This analysis reveals that complicated smear signatures can occur under these circumstances, even for the special case in which the radar moves with constant speed along a level flight path while collecting SAR measurement data using a broadside imaging geometry. This analysis considers such range migration effects for spotlight-mode SAR [7], [8].

A thorough investigation of the morphology of mover smears for general maneuvering targets is given in [9]. This analysis utilizes stationary phase techniques applied to local subaperture SAR images in order to generate general analytic equations for the shape of surface mover smears. Specifically, this study analyzes spotlight SAR for broadside imaging geometry and straight and level radar flight path in order to compute analytic nonparametric expressions for smear signature shapes for surface targets with arbitrary motion profiles. It is possible that this analysis could yield additional insight into the target motion characteristics based upon the details of these smear signatures within SAR imagery.

The fundamental analytics in the previous analysis [9] is based upon the development of analytic equations for the smear signature induced by a moving target within SAR subaperture images [10], [11], [12]. The structure of the full-aperture SAR image can be inferred from the details of the sub-aperture images, since the concatenation of the smaller smears of the sub-aperture images 
yields the full-aperture smear. The previous analysis [9] demonstrates the viability and utility of the predictive signature equations for a number of special cases. One of these examples is the case of a surface target that is moving with a constant heading while transitioning from a higher speed to a lower speed. The current analysis examines the effect of varying the rate at which the speed decreases.

\section{IMAGE FORMATION}

The radar transmits and receives a waveform for each point along the synthetic aperture. The phase history data are measured at the various frequencies $f_{m^{\prime}}$ spanned by the radar waveforms and at the different times $t$ along the synthetic aperture. These operations give the downconverted, frequency-domain SAR measurement data in the original "polar" format. The polarformatted frequency-domain data are related to collected phase history data by a one-dimensional (1-D) Fourier transform along the range dimension. A polar-to-rectangle sampling operation [7] can be applied to obtain the Cartesian-sampled data. Then, a 2D discrete Fourier transform (DFT) gives the desired spotlight-mode SAR image.

\section{Signature Predictions}

Ref. [9] applies the techniques of stationary phase to sub-aperture images in order to derive general expressions for the smear signature for a surface target moving with an arbitrary motion profile. The following true target trajectory is considered [9]:

$$
\begin{aligned}
\alpha(t) & =\bar{\alpha}_{0}+\cos \left(\bar{\phi}_{0}\right)\left\{\bar{v}_{0} t\right. \\
& \left.+\bar{w}_{0} \bar{\gamma}_{0} \ln \left(\cosh \left(\frac{t-\bar{t}_{0}}{\bar{\gamma}_{0}}\right)\right)\right\}, \\
\beta(t) & =\bar{\beta}_{0}+\sin \left(\bar{\phi}_{0}\right)\left\{\bar{v}_{0} t\right. \\
& \left.+\bar{w}_{0} \bar{\gamma}_{0} \ln \left(\cosh \left(\frac{t-\bar{t}_{0}}{\bar{\gamma}_{0}}\right)\right)\right\},
\end{aligned}
$$

in terms of the free parameters $\left\{\bar{\alpha}_{0}, \bar{\beta}_{0}, \bar{v}_{0}, \bar{w}_{0}, \bar{\gamma}_{0}, \bar{\phi}_{0}, \bar{t}_{0}\right\}$. The current analysis examines the effects of varying the rate of target braking, as modeled by the parameter $\bar{\gamma}_{0}$. These equations model a target that is moving at a higher speed and then performs a braking maneuver, before finally settling at a lower speed.

The detailed analytics of [9] yield the following form for the predicted SAR signature of a braking target, expressed in terms of the down-range and cross-range components:

$$
\begin{aligned}
x\left(\tau_{s}\right) & =\bar{\alpha}_{0}-\tau_{s}^{2} \sin \left(\bar{\phi}_{0}\right) \frac{\bar{v}_{0}}{\kappa_{0}} \\
& +\cos \left(\bar{\phi}_{0}\right) \bar{w}_{0} \bar{\gamma}_{0} \ln \left(\cosh \left(\frac{\tau_{s}-\bar{t}_{0}}{\bar{\gamma}_{0}}\right)\right) \\
& -\tau_{s} \bar{w}_{0}\left\{\cos \left(\bar{\phi}_{0}\right)\right. \\
& \left.+\frac{\tau_{s}}{\kappa_{0}} \sin \left(\bar{\phi}_{0}\right)\right\} \tanh \left(\frac{\tau_{s}-\bar{t}_{0}}{\bar{\gamma}_{0}}\right), \\
y\left(\tau_{s}\right) & =\bar{\beta}_{0}+\bar{v}_{0}\left\{\kappa_{0} \cos \left(\bar{\phi}_{0}\right)+\tau_{s} \sin \left(\bar{\phi}_{0}\right)\right\} \\
& +\sin \left(\bar{\phi}_{0}\right)\left\{\bar{v}_{0} \tau_{s}\right. \\
& \left.+\bar{w}_{0} \bar{\gamma}_{0} \ln \left(\cosh \left(\frac{\tau_{s}-\bar{t}_{0}}{\bar{\gamma}_{0}}\right)\right)\right\} \\
& +\bar{w}_{0}\left\{\kappa_{0} \cos \left(\bar{\phi}_{0}\right)\right. \\
& \left.+\tau_{s} \sin \left(\bar{\phi}_{0}\right)\right\} \tanh \left(\frac{\tau_{s}-\bar{t}_{0}}{\bar{\gamma}_{0}}\right) .
\end{aligned}
$$

\section{Signature Results}

This section examines the specific signatures that result for the case of a moving surface target undergoing a braking maneuver. This decrease in the target speed is modeled through the use of a hyperbolic tangent speed profile as provided in (1)(2). For the first example shown in Figure 1, the parameter values are selected to be $\left\{\bar{\alpha}_{0}=0.0 \mathrm{~m}\right\}$, $\left\{\bar{\beta}_{0}=0.0 \mathrm{~m}\right\},\left\{\bar{v}_{0}=8 \mathrm{~m} / \mathrm{s}\right\},\left\{\bar{w}_{0}=-4 \mathrm{~m} / \mathrm{s}\right\}$, $\left\{\bar{\gamma}_{0}=0.5 \mathrm{~s}\right\}$, and $\left\{\bar{\phi}_{0}=-80.9^{\circ}\right\}$. This set of target motion parameters is consistent with the desired case in which a constant-heading target is initially moving with approximately constant speed and then undergoes a braking maneuver (i.e., a sudden decrease in target speed), and then settles to a lower approximately constant speed with the same heading.

Figures 2 and 3 present the SAR smears for the case of a mainbeam that is pointed off of the port 


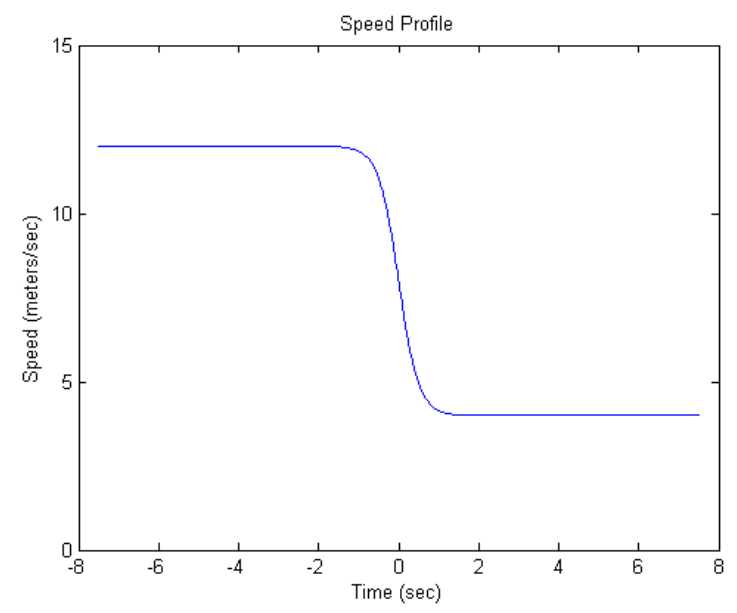

Fig. 1. Speed profile for a target that is undergoing a braking maneuver according to the motion parameters $\left\{\bar{\alpha}_{0}=0.0 \mathrm{~m}\right\}$, $\left\{\bar{\beta}_{0}=0.0 \mathrm{~m}\right\},\left\{\bar{v}_{0}=8 \mathrm{~m} / \mathrm{s}\right\},\left\{\bar{w}_{0}=-4 \mathrm{~m} / \mathrm{s}\right\},\left\{\bar{\gamma}_{0}=0.5 \mathrm{~s}\right\}$, and $\left\{\bar{\phi}_{0}=-80.9^{\circ}\right\}$

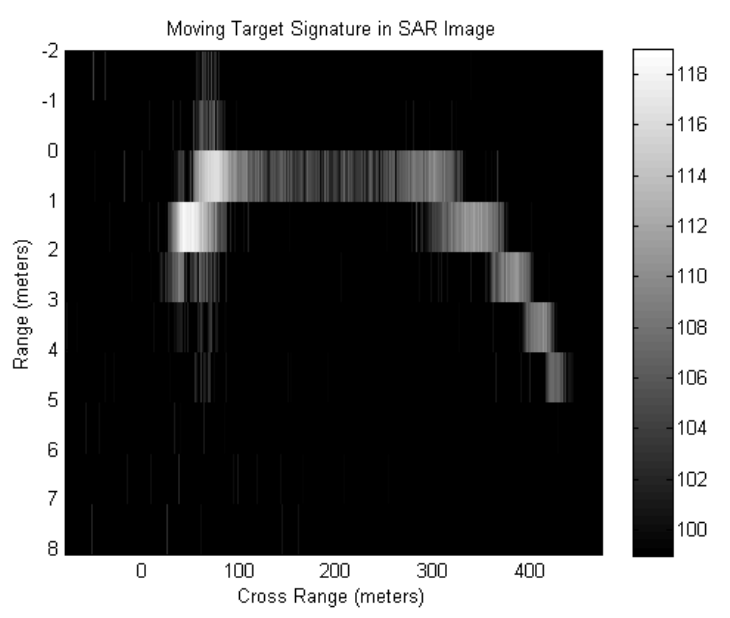

Fig. 2. Resulting target signature with $\left\{\bar{\alpha}_{0}=0.0 \mathrm{~m}\right\}$, $\left\{\bar{\beta}_{0}=0.0 \mathrm{~m}\right\},\left\{\bar{v}_{0}=8 \mathrm{~m} / \mathrm{s}\right\},\left\{\bar{w}_{0}=-4 \mathrm{~m} / \mathrm{s}\right\},\left\{\bar{\gamma}_{0}=0.5 \mathrm{~s}\right\}$, and $\left\{\bar{\phi}_{0}=-80.9^{\circ}\right\}$ for the case of leftward pointing radar mainbeam

(i.e., left) side of the radar platform. In contrast, Figures 4 and 5 compare the actual and predicted SAR smear signatures for the case of an identical braking target as with the previous example, but with the radar mainbeam is pointed off of the starboard (i.e., right) side of the radar platform. Again, there is excellent agreement between the predicted and actual SAR signatures in terms of smear position, extent, and shape. This case gives a different signature which almost crosses itself, which is quite different form the typical bowed signatures.

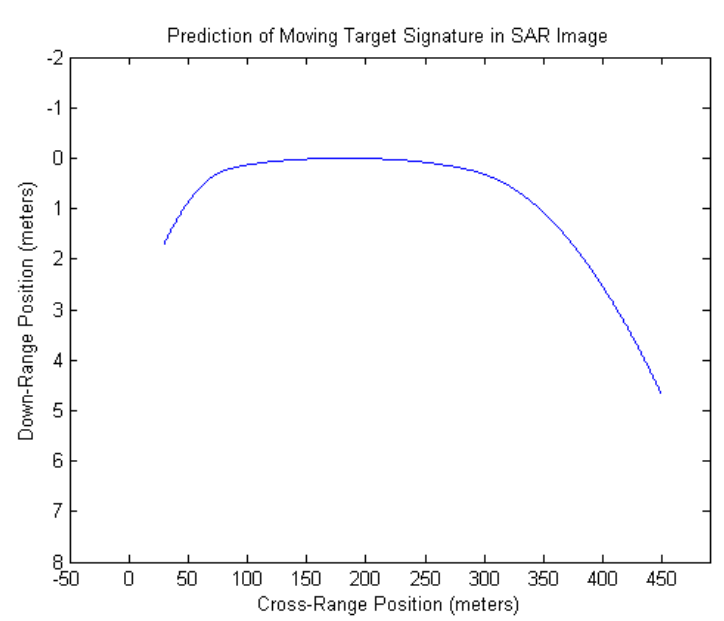

Fig. 3. Corresponding predicted target signature based upon the true target motion for the case of $\left\{\bar{\alpha}_{0}=0.0 \mathrm{~m}\right\},\left\{\bar{\beta}_{0}=\right.$ $0.0 \mathrm{~m}\},\left\{\bar{v}_{0}=8 \mathrm{~m} / \mathrm{s}\right\},\left\{\bar{w}_{0}=-4 \mathrm{~m} / \mathrm{s}\right\},\left\{\bar{\gamma}_{0}=0.5 \mathrm{~s}\right\}$, and $\left\{\bar{\phi}_{0}=-80.9^{\circ}\right\}$

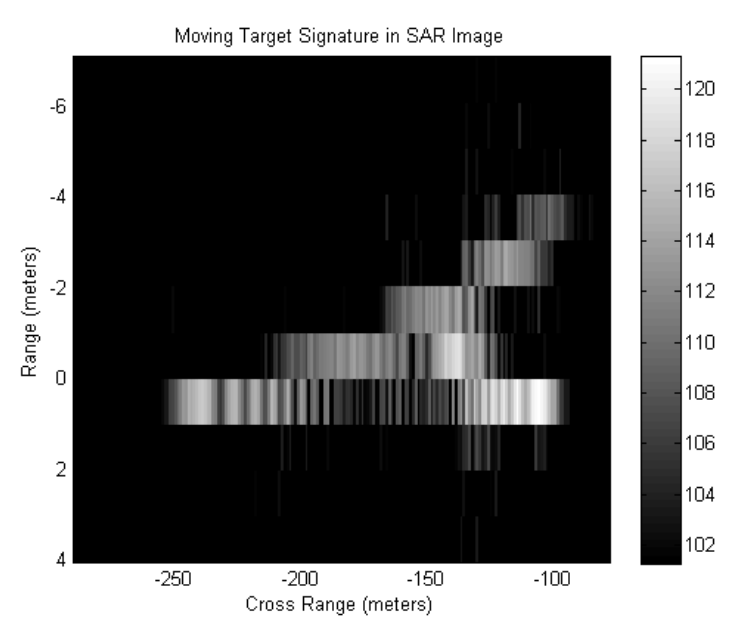

Fig. 4. Target signature in spotlight SAR imagery for a target with parameters $\left\{\bar{\alpha}_{0}=0.0 \mathrm{~m}\right\},\left\{\bar{\beta}_{0}=0.0 \mathrm{~m}\right\},\left\{\bar{v}_{0}=8 \mathrm{~m} / \mathrm{s}\right\}$, $\left\{\bar{w}_{0}=-4 \mathrm{~m} / \mathrm{s}\right\},\left\{\bar{\gamma}_{0}=0.5 \mathrm{~s}\right\}$, and $\left\{\bar{\phi}_{0}=-80.9^{\circ}\right\}$ for the case of rightward pointing radar mainbeam

The next example of Figure 6 considers a target that is undergoing a gentler braking maneuver, as modeled with the parameter set: $\left\{\bar{\alpha}_{0}=0.0 \mathrm{~m}\right\}$, $\left\{\bar{\beta}_{0}=0.0 \mathrm{~m}\right\},\left\{\bar{v}_{0}=8 \mathrm{~m} / \mathrm{s}\right\},\left\{\bar{w}_{0}=-4 \mathrm{~m} / \mathrm{s}\right\}$, $\left\{\bar{\gamma}_{0}=2.5 \mathrm{~s}\right\}$, and $\left\{\bar{\phi}_{0}=-80.9^{\circ}\right\}$. Only the parameter $\left\{\bar{\gamma}_{0}=2.5 \mathrm{~s}\right\}$ has a different value. Figures 7 and 8 present the SAR smears for the case of a radar mainbeam that is pointed off of the port (i.e., left) side of the radar platform.

Figures 9 and 10 compare the actual and predicted SAR smear signatures for the case of a more 


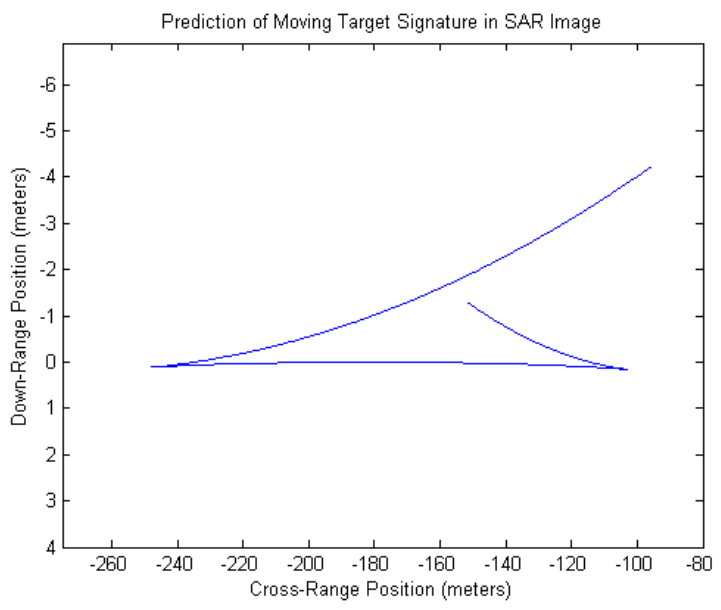

Fig. 5. Corresponding predicted signature for a target with $\left\{\bar{\alpha}_{0}=0.0 \mathrm{~m}\right\},\left\{\bar{\beta}_{0}=0.0 \mathrm{~m}\right\},\left\{\bar{v}_{0}=8 \mathrm{~m} / \mathrm{s}\right\},\left\{\bar{w}_{0}=\right.$ $-4 \mathrm{~m} / \mathrm{s}\},\left\{\bar{\gamma}_{0}=0.5 \mathrm{~s}\right\}$, and $\left\{\bar{\phi}_{0}=-80.9^{\circ}\right\}$ for the case of a rightward pointing radar mainbeam

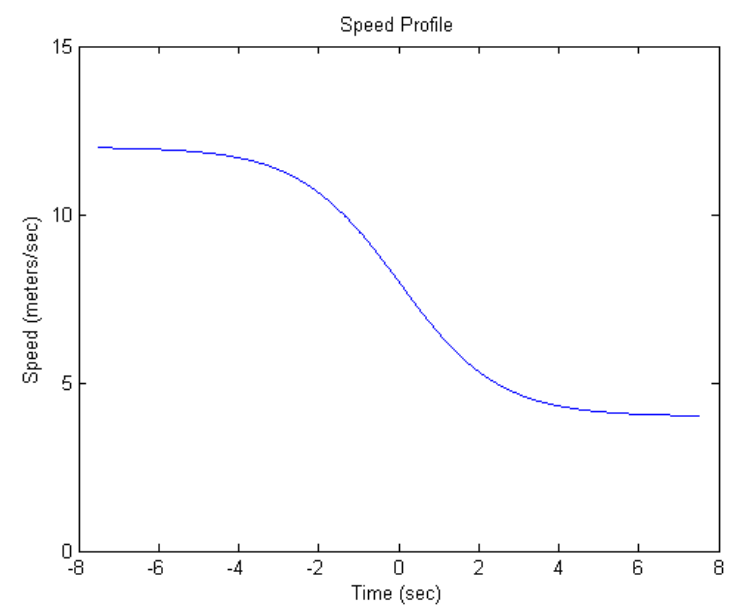

Fig. 6. Speed profile for a target that is undergoing a braking maneuver according to the motion parameters $\left\{\bar{\alpha}_{0}=0.0 \mathrm{~m}\right\}$, $\left\{\bar{\beta}_{0}=0.0 \mathrm{~m}\right\},\left\{\bar{v}_{0}=8 \mathrm{~m} / \mathrm{s}\right\},\left\{\bar{w}_{0}=-4 \mathrm{~m} / \mathrm{s}\right\},\left\{\bar{\gamma}_{0}=2.5 \mathrm{~s}\right\}$, and $\left\{\bar{\phi}_{0}=-80.9^{\circ}\right\}$

slowly braking target as with the previous example, but with the radar mainbeam is pointed off of the starboard (i.e., right) side of the radar platform. Again, there is excellent agreement between the predicted and actual SAR signatures in terms of smear position, extent, and shape. This case gives a different signature which almost crosses itself, just as for the case of a braking target with a larger value for the acceleration in the direction opposite to that of the direction of motion.

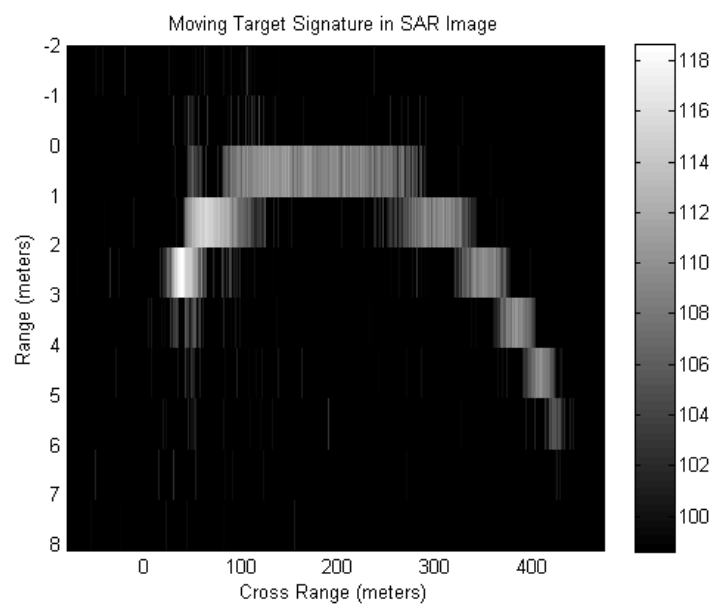

Fig. 7. Resulting target signature with $\left\{\bar{\alpha}_{0}=0.0 \mathrm{~m}\right\}$, $\left\{\bar{\beta}_{0}=0.0 \mathrm{~m}\right\},\left\{\bar{v}_{0}=8 \mathrm{~m} / \mathrm{s}\right\},\left\{\bar{w}_{0}=-4 \mathrm{~m} / \mathrm{s}\right\},\left\{\bar{\gamma}_{0}=2.5 \mathrm{~s}\right\}$, and $\left\{\bar{\phi}_{0}=-80.9^{\circ}\right\}$ for the case of leftward pointing radar mainbeam

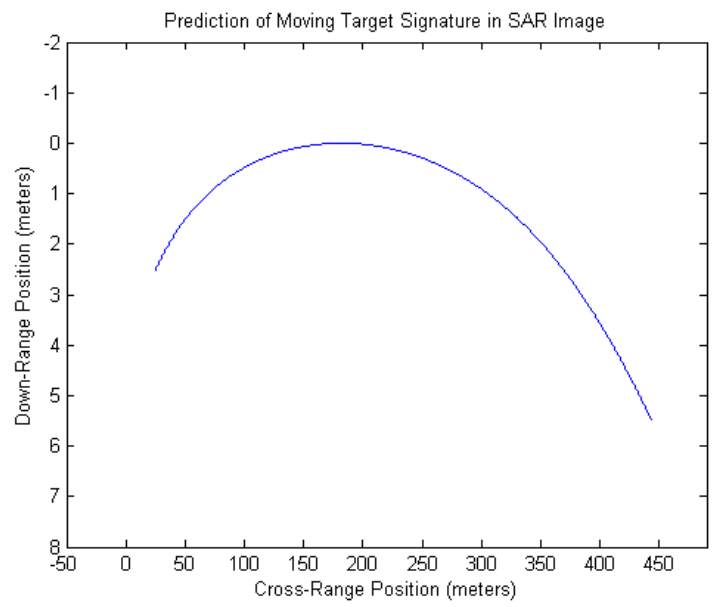

Fig. 8. Predicted target signature based upon the true target motion for the case of $\left\{\bar{\alpha}_{0}=0.0 \mathrm{~m}\right\},\left\{\bar{\beta}_{0}=0.0 \mathrm{~m}\right\},\left\{\bar{v}_{0}=\right.$ $8 \mathrm{~m} / \mathrm{s}\},\left\{\bar{w}_{0}=-4 \mathrm{~m} / \mathrm{s}\right\},\left\{\bar{\gamma}_{0}=2.5 \mathrm{~s}\right\}$, and $\left\{\bar{\phi}_{0}=-80.9^{\circ}\right\}$

\section{CONCLUSION}

This paper has examined the signatures of surface moving targets that are undergoing braking maneuvers. Consistent signatures smears were obtained from both the application of analytic predictive equations and the generation using SAR simulations. It was found that the resulting signature shapes need not conform to conventional bowshaped morphologies. In particular, this analysis compared signature of targets that are braking quickly versus those that are braking more slowly. 


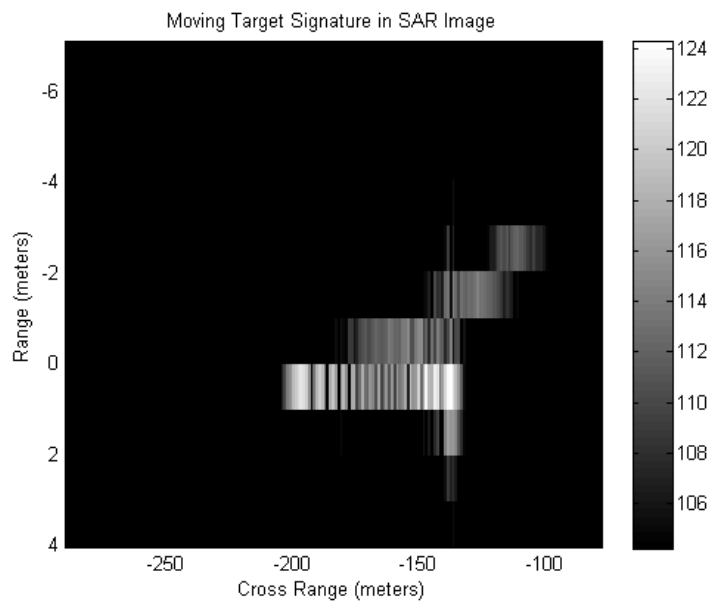

Fig. 9. Target signature in spotlight SAR imagery for a target with parameters $\left\{\bar{\alpha}_{0}=0.0 \mathrm{~m}\right\},\left\{\bar{\beta}_{0}=0.0 \mathrm{~m}\right\},\left\{\bar{v}_{0}=8 \mathrm{~m} / \mathrm{s}\right\}$, $\left\{\bar{w}_{0}=-4 \mathrm{~m} / \mathrm{s}\right\},\left\{\bar{\gamma}_{0}=2.5 \mathrm{~s}\right\}$, and $\left\{\bar{\phi}_{0}=-80.9^{\circ}\right\}$ for the case of rightward pointing radar mainbeam

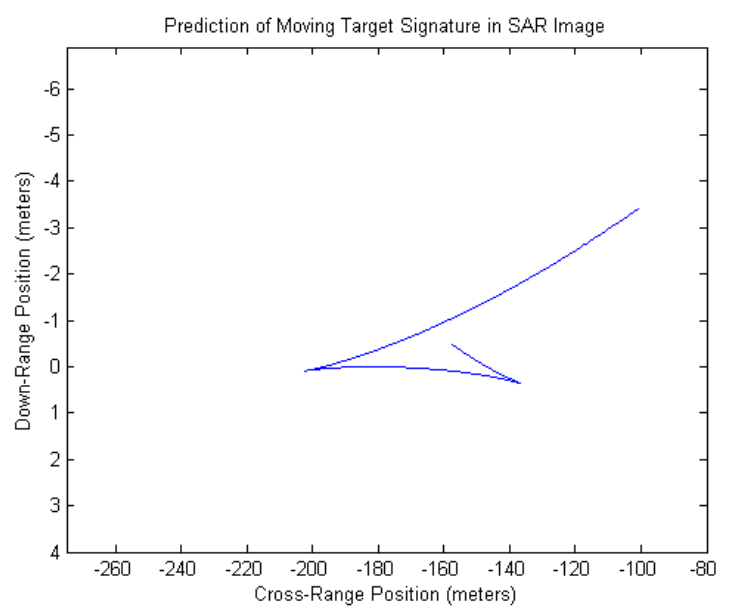

Fig. 10. Corresponding predicted signature for a target with $\left\{\bar{\alpha}_{0}=0.0 \mathrm{~m}\right\},\left\{\bar{\beta}_{0}=0.0 \mathrm{~m}\right\},\left\{\bar{v}_{0}=8 \mathrm{~m} / \mathrm{s}\right\}$, $\left\{\bar{w}_{0}=-4 \mathrm{~m} / \mathrm{s}\right\},\left\{\bar{\gamma}_{0}=2.5 \mathrm{~s}\right\}$, and $\left\{\bar{\phi}_{0}=-80.9^{\circ}\right\}$ for the case of a rightward pointing radar mainbeam

\section{ACKNOWLEDGMENT}

DoD Distribution Statement A: Unlimited Distribution. The views expressed in this document are those of the authors and do not reflect the official policy or position of the Department of Defense or the U.S. Government.

\section{REFERENCES}

[1] R. P. Perry, R. C. DiPietro, and R. L. Fante, "SAR imaging of moving targets," IEEE Transactions on Aerospace and Electronic Systems, vol. 35, no. 1, pp. pp. 188-200, Jan 1999.
[2] R. K. Raney, "Synthetic aperture imaging radar and moving targets," IEEE Transactions on Aerospace and Electronic Systems, vol. 7, no. 3, pp. pp. 499-505, May 1971.

[3] R. H. Stolt, "Migration by fourier transform," Geophysics, vol. 43, pp. pp. 23-48, 1978.

[4] S. Rahman, "Focusing moving targets using range migration algorithm in ultra wideband low frequency synthetic aperture radar," Master's thesis, Blekinge Institue of Technology, Jun 2010.

[5] J. K. Jao, "Theory of synthetic aperture radar imaging of a moving target," IEEE Transactions on Geoscience and Remote Sensing, vol. 39, no. 9, pp. 1984 -1992, Sep 2001.

[6] D. Garren, J. W. Scrofani, M. Tummala, and J. C. McEachen, "Target migration path morphology of moving targets in spotlight sar," Proc. of SPIE Vol. 8746 87460B-7: "Algorithms for Synthetic Aperture Radar Imagery XX" of the SPIE Defense, Security, and Sensing Conference held 29 April - 3 May 2013 in Baltimore, Maryland; USA, May 2013.

[7] C. V. Jakowatz Jr., D. E. Wahl, P. H. Eichel, D. C. Ghiglia, and P. A.Thompson, Spotlight-Mode Synthetic Aperture Radar: A Signal Processing Approach. Norwell, MA, USA: Kluwer Academic Publishers, 1996.

[8] W. G. Carrara, R. S. Goodman, and R. M. Majewski, Spotlight Synthetic Aperture Radar Signal Processing Algorithms. Norwood, MA, USA: Artech House, 1995.

[9] D. A. Garren, "Smear signature morphology of surface targets with arbitrary motion in spotlight synthetic aperture radar imagery," IET Radar, Sonar and Navigation, vol. 8, no. 5, pp. 435-448, Jun 2014.

[10] J. Zhang, J. Xu, Y. Peng, and X. Wang, "Speckle filtering of SAR images based on sub-aperture technique and principal component analysis," Communications and Information Technology 2005. ISCIT 2005. IEEE International Symposium on, vol. 2, pp. 1217 - 1222, Oct 2005.

[11] M. Soumekh, "A system model and inversion for synthetic aperture radar imaging," IEEE Transactions on Image Processing, vol. 1, no. 1, pp. pp. 64-76, Jan 1992.

[12] _ - "Digital spotlighting and coherent subaperture image formation for stripmap synthetic aperture radar," Image Processing, 1994, Proceedings, ICIP-94, IEEE International Conference, vol. 1, pp. pp. 476-480, Nov 1994. 